\title{
Trabajo colaborativo y estrategias de aprendizaje en entornos virtuales en jóvenes universitarios
}

Collaborative work and learning strategies in virtual environments in young University students

Estratégias de trabalho e de aprendizagem colaborativa em ambientes virtuais em Young University

DOI: http://dx.doi.org/10.23913/ride.v7i14.274

René Rodríguez Zamora

Universidad Autónoma de Sinaloa, México rene.rodriguez@info.uas.edu.mx

Leonor Antonia Espinoza Núñez

Universidad Autónoma de Sinaloa, México leonor_espinoza63@hotmail.com

\section{Resumen}

El presente artículo se deriva de un proyecto integral acerca de la creación de espacios virtuales basados en el estudio de la psicología de la educación virtual; en este sentido, se analizó la relación que existe entre el trabajo colaborativo y las estrategias de aprendizaje utilizadas por los jóvenes para su aprovechamiento en entornos virtuales. Se trató de identificar las orientaciones o preferencias para la realización de trabajo colaborativo en la generación del aprendizaje autónomo, así como las estrategias utilizadas en el desempeño de los estudiantes al contacto con los entornos virtuales como herramientas pedagógicas. El enfoque metodológico utilizado fue cuantitativo de tipo ex post-facto con un nivel de alcance descriptivo de las unidades de análisis: trabajo colaborativo y estrategias de aprendizaje, tomando como muestra de estudio sujetos del nivel medio superior y superior de entre 15 y 23 años de edad del estado de Sinaloa, México. 
Se encontró que los estudiantes de bachillerato a pesar de mostrar más facilidad para la búsqueda de información, carecen de iniciativa para la búsqueda de estrategias autónomas, mostrando dificultad para discriminar y seleccionar la información más adecuada como parte de un proceso de análisis. Por su parte, los jóvenes de nivel superior mostraron disposición para trabajar en equipo y mayor habilidad para la búsqueda de información, sin embargo, se reportaron con dificultades para el manejo de plataformas virtuales debido a que perciben que no hay participación por parte del docente. Ambas poblaciones reconocieron contar con ciertas destrezas para el aprendizaje autónomo y el desempeño del trabajo colaborativo, sin embargo la percepción que manifestaron es que el desarrollo de estas competencias no se ha potenciado lo suficiente. Los resultados obtenidos podrán servir como pauta para futuras investigaciones orientadas a la generación de propuestas formativas en el marco de entornos virtuales.

Palabras clave: Trabajo colaborativo, estrategias de aprendizaje, entornos virtuales, tecnologías de la información y comunicación.

\section{Abstract}

This article is derived from a comprehensive project on creating virtual spaces based on the study of the psychology of virtual education; in this sense, discussed the relationship between collaborative work and learning strategies used by young people for use in virtual environments. The aim was to identify the guidelines or preferences for the realization of collaborative work in the generation of autonomous learning, as well as the strategies used in the performance of the students in contact with virtual environments as pedagogical tools. The methodological approach used was quantitative type ex post-facto with a level of descriptive scope of units of analysis: collaborative work and learning strategies, taking as sample of study subjects of level between superior and superior between 15 and 23 years of age of the State of Sinaloa, Mexico.

Key words: Collaborative work, learning strategies, virtual environments, Information and Communication Technology (ICT). 


\section{Resumo}

Este artigo é derivado de um projeto abrangente sobre a criação de espaços virtuais com base no estudo da psicologia da educação virtual; Neste sentido, a relação entre o trabalho e as estratégias de aprendizagem colaborativa utilizados pelos jovens para a sua utilização em ambientes virtuais analisados. Foi para identificar orientações ou preferências para a realização de trabalho colaborativo na geração de aprendizagem autónoma e as estratégias utilizadas no desempenho dos estudantes em contato com ambientes virtuais como ferramentas de ensino. A abordagem metodológica utilizada foi ex quantitativa post-facto com um nível de alcance descritivo das unidades de análise: estratégias de trabalho e de aprendizagem colaborativa, tomando como amostra do estudo submete a média superior e de nível superior entre 15 e 23 anos velho estado de Sinaloa, no México.

Verificou-se que os estudantes do ensino médio, apesar de mostrar mais facilmente procurar informações, falta de iniciativa para as estratégias de busca autónomas, mostrando dificuldade em discriminar e selecionar a informação mais adequada como parte de um processo de análise. Enquanto isso, o nível superior jovem mostrou disposição para o trabalho em equipe e uma maior capacidade de busca de informações, no entanto, relataram dificuldades em gerir plataformas virtuais, porque eles percebem que não há participação do professor. Ambas as populações reconhecidos ter certas habilidades de aprendizagem independente e desempenho no trabalho colaborativo, no entanto, expressa a percepção é que o desenvolvimento destas habilidades não foi promovido o suficiente. Os resultados obtidos podem servir como um guia para futuras pesquisas destinadas a gerar propostas de formação em ambientes virtuais.

Palavras-chave: trabalho colaborativo, estratégias de aprendizagem, ambientes virtuais, tecnologia da informação e comunicação.

Fecha Recepción: Junio $2016 \quad$ Fecha Aceptación: Enero 2017 


\section{Introducción}

Dentro del enfoque tradicionalista de la educación, el profesor como figura central es el principal responsable del aprendizaje del estudiante, pues moldeado en un sistema cerrado y lineal es el encargado de la selección y diseño de lo que se aprende, y del cómo se aprende. Así mismo, la valoración de los logros está en función de los productos y su calidad. Con la teoría constructivista, bajo una perspectiva social y un enfoque activo, el joven universitario se centra en el proceso de construcción de los saberes a partir de la interacción, no solo del maestro con el alumno, sino de la interacción entre los miembros del grupo, consolidando grupos o comunidades de aprendizaje. En esta perspectiva existe un mundo real que experimentamos pero el significado es impuesto en el mundo por nosotros, por ello, para Gross (2002) se entiende la enseñanza como un proceso que no se centra en la transmisión de la información al alumno, sino que debe focalizarse en el desarrollo de habilidades para construir y reconstruir conocimientos en respuesta a la demanda de un determinado contexto o situación.

Este nuevo enfoque hace que se requiera un nuevo rol para la figura del profesor pues deja de ser por un lado el actor central y por otro sus actividades cambian de ser directivas a ser orientadoras y mediadoras, cuya función principal cambia de ser instructora a ser promotora de ambientes de aprendizaje. En este sentido, el trabajo colaborativo exige que los miembros del grupo compartan las tareas y las aportaciones para un objetivo en común. Alcalde (2015) lo explica como un proceso en el cual cada individuo aprende más de lo que aprendería por sí solo, fruto de la interacción de los integrantes del equipo, y por lo tanto, un trabajo hecho en un grupo de forma colaborativa, tiene un resultado más enriquecedor que el que tendría la suma del trabajo individual.

La interacción implica entonces una serie de actividades mediadoras a realizar entre los participantes para el logro del objetivo, pues las aportaciones no solo se suman, sino que desde la búsqueda de información, se organiza, se selecciona, entra además en un proceso de retroalimentación mutua donde se discute, se negocian las diferentes apreciaciones en la construcción del conocimiento. De esta forma, compartir el entendimiento y la comprensión es el arte que el profesor tendrá que fomentar en el clima del aula y fuera de ella. Jhonson 
(1999) habla de habilidades mixtas cuando explica la función del trabajo colaborativo, es decir implica tanto características del desarrollo personal como de carácter social, donde cada sujeto aporta mediante el diálogo como principal medio, su propia capacidad de prospección, misma que en la intermediación tendrá la posibilidad de reconstruirse. Roinstein (2006) habla de una visión compartida donde cada miembro toma en cuenta lo que el otro comprende, en este sentido la flexibilidad y la apertura son cualidades que se muestran mediante el intercambio y el diálogo, desde esta condición el error y el conflicto deja de ser un obstáculo y se convierte en sí mismo en experiencia de aprendizaje.

Por otra parte, las características del mundo globalizado, exigen una rápida adaptación a las demandas en diversos estilos y formas de vida que nos conducen a nuevas formas de interacción. Los avances en las Tecnologías de la Información y su utilidad en los diferentes ámbitos, han producido en las nuevas generaciones una cultura social con patrones de convivencia muy particulares. Los jóvenes llevan al espacio educativo esa nueva dimensión tecnológica que de no ser aprovechada por los profesores, puede convertirse en distractor y no en una herramienta de utilización pedagógica.

Las herramientas tecnológicas mediante la creación de entornos virtuales representan una estrategia pedagógica que facilita la interacción, ya que promueve el aprendizaje simultáneo y cooperativo a pesar de las limitaciones de la distancia y la permanencia, que se han convertido cada vez más en una barrera que descarta la habilidad individual en condiciones específicas. Es por ello que los resultados de este estudio generan una reflexión de la práctica educativa en el uso de estrategias aprovechables en entornos virtuales, considerando que estos entornos no se crean de manera automática y natural, han de crearse como parte de los ambientes de aprendizaje, y para ello es importante identificar el uso común de estrategias de aprendizaje y las preferencias y estilos que el joven emplea para el aprendizaje colaborativo en su acercamiento a los entornos virtuales.

El objetivo de este estudio consistió en identificar las características de las estrategias de aprendizaje utilizadas por los jóvenes de nivel medio y superior, así como su acercamiento al trabajo colaborativo en entornos virtuales. 


\section{Los entornos virtuales como alternativa de aprendizaje}

Las teorías y enfoques educativos se han venido transformando conforme al desarrollo de la sociedad y la manera en que su esfera productiva va marcando nuevos parámetros, de tal forma que las metas educativas en cuanto a la formación de los estudiantes en sus diferentes disciplinas han tenido que amoldarse paralelamente a este vertiginoso cambio de la sociedad a la que se debe el hombre. Se exige así un individuo capaz no solo de reproducir esquemas, sino de desenvolverse con habilidad en los nuevos modelos que la tecnología va introduciendo, y de producir a partir de ello las innovaciones que los problemas sociales van demandando. Es importante así que lo aprendido sirva de punto de partida para el análisis de las situaciones que enfrenta, y oriente sus competencias a la búsqueda de soluciones.

A partir de este escenario es urgente que los actores educativos se centren en la construcción de ambientes de aprendizaje que les permitan desarrollar tales requerimientos, en este tenor, las herramientas tecnológicas proporcionan el beneficio de ir acorde y al ritmo del desarrollo del mundo globalizado, por lo que el desfase en épocas deja de ser un riesgo latente, y por otra parte, proporcionan la organización de la información digital al alcance y con ello la posibilidad de la interacción con el mundo, eliminando los contratiempos que la distancia contrapone al contacto presencial; en el ámbito del aprendizaje abre la ventana a un nuevo formato en el que la autonomía y la autodirección se convierten en la forma idónea para administrar el proceso cognitivo.

Sin embargo, este camino no viene trazado desde la tecnología en sí, sino que para que sea una herramienta pedagógica, su diseño debe estar respaldado en las teorías del aprendizaje que de alguna forma retoman la dimensión de lo virtual. Breansford (et al., 1999) señala entre los principales aspectos para que se generen estos nuevos ambientes de aprendizaje, la necesidad de promover cambios en las metas educativas, y entender que los ambientes deberán ir centrados en quien aprende y no en quien enseña, en el proceso de adquisición y no en la permanencia del conocimiento, y en el terreno de la evaluación como la valoración de los mecanismos y la retroalimentación, y no en el producto, ello implica ver la educación como un proceso de formación y no de información, en la conformación de comunidades de aprendizaje basados en la socialización mediatizada del saber, en lugar de la conducción lineal centrada en el que más sabe. La integración de estas nuevas perspectivas requiere de 
toda una sistematización que tome en cuenta la innovación de los medios digitales, de esta manera, la interacción mediada en los medios virtuales mediante las bondades de los alcances tecnológicos representa una oportunidad. Un entorno virtual es un ambiente de aprendizaje basado en medios digitales donde la interacción adquiere diferentes matices, ya que puede ser síncrono o asíncrono, es un todo organizado para que confluyan en su uso un conjunto de sujetos en la construcción de los saberes. Bello Díaz (2005) llama a los entornos virtuales para el aprendizaje "aulas sin paredes" y afirma que es un espacio social virtual, cuyo mejor exponente actual es Internet, no es presencial, sino representacional, no es proximal, sino distal, no es sincrónico, sino multicrónico, y no se basa en recintos espaciales con interior, frontera y exterior, sino que depende de redes electrónicas cuyos nodos de interacción pueden estar diseminados por diversos países.

Considerando el aprendizaje como proceso psicológico, el entorno virtual conlleva algunas ventajas. Ballenato (2009) destaca entre ellos la motivación, el aprendizaje activo y cooperativo, la autodirección del estudio y la autoevaluación, ya que el estudiante establece sus propios objetivos y planifica las actividades en función de la posibilidad de sus recursos.

\section{Método}

Para la aplicación del estudio de campo se tomó como segmentos poblacionales a estudiantes de nivel medio superior y superior cuyas edades oscilan entre los 15 y 23 años de edad. Con una muestra representativa de 150 sujetos de nivel económico medio con acceso a servicios de conexión a Internet, seleccionados aleatoriamente de diversas instituciones educativas de la zona urbana del municipio de Mazatlán, Sinaloa, México.

Para la recolección de datos se diseñaron 2 cuestionarios a partir de dos unidades de análisis principales: trabajo colaborativo y estrategias de aprendizaje en entornos virtuales. Este instrumento de recolección de datos fue validado aplicando el coeficiente Alfa de Cronbach. Los cuestionarios están compuestos por 27 y 37 items respectivamente abordando los principales atributos de las variables a medir.

El enfoque metodológico utilizado fué cuantitativo de tipo ex post-facto (Bizquerra, 2004) con un nivel de alcance descriptivo de las unidades de análisis: trabajo colaborativo y estrategias de aprendizaje. Para calcular el tamaño de la muestra se utilizó la siguiente 
ecuación aplicable para realizar análisis estadístico para estudios descriptivos en los cuales las unidades de análisis se manejan siguiendo el enfoque cuantitativo:

$$
n=\frac{N Z^{2} S^{2}}{d^{2}(N-1)+Z^{2} S^{2}}
$$

\section{Resultados}

\section{El aprendizaje autodirigido y el trabajo colaborativo en entornos virtuales}

En el estudio realizado se presenta la agrupación de variables que describen las características con las que el estudiante desarrolla las competencias para el trabajo autodirigido y el aprendizaje colaborativo, es decir, refieren a la habilidad del estudiante de nivel medio superior y superior para orientar su aprendizaje con iniciativa, detectando sus necesidades, formulando sus objetivos, identificando sus propios recursos, y sus preferencias para el desempeño en el trabajo colaborativo utilizando los espacios o entornos virtuales.

Tabla 1. Características del aprendizaje autodirigido en adolescentes de bachillerato.

\begin{tabular}{|l|c|c|c|c|c|c|}
\hline \multicolumn{1}{|c|}{ VARIABLE } & MIN & MAX & X & S & CV & MO \\
\hline Necesidad de información & 1 & 10 & 7.82 & 2.13 & 0.27 & 8 \\
\hline Metas al estudiar & 0 & 10 & 8.06 & 2.28 & 0.28 & 10 \\
\hline Elección de procedimientos & 5 & 10 & 8.49 & 1.43 & 0.17 & 10 \\
\hline Selección de estrategias & 0 & 10 & 8.49 & 0 & 0 & 0 \\
\hline Elección de momentos óptimos & 1 & 10 & 8.02 & 1.70 & 0.21 & 9 \\
\hline Utilidad del aprendizaje & 1 & 10 & 8.57 & 1.88 & 0.22 & 10 \\
\hline Selección de recursos & 2 & 10 & 8.41 & 1.80 & 0.21 & 9 \\
\hline Autoevaluación & 2 & 10 & 8.22 & 1.79 & 0.22 & 9 \\
\hline Mejora del desempeño & 0 & 10 & 8.31 & 1.91 & 0.23 & 10 \\
\hline Iniciativa & 0 & 10 & 7.53 & 2.36 & 0.31 & 9 \\
\hline Aprendizaje mediante las TIC & 0 & 10 & 7.90 & 2.04 & 0.26 & 9 \\
\hline Expectativa & 3 & 10 & 7.98 & 1.77 & 0.22 & 8 \\
\hline \multicolumn{1}{|c|}{ Xx=8.17 } & Ls= 8.50 & Li= 7.84 & & \\
\hline
\end{tabular}

Fuente: Elaboración propia. 
Figura 1. Características del aprendizaje autodirigido en estudiantes de bachillerato.

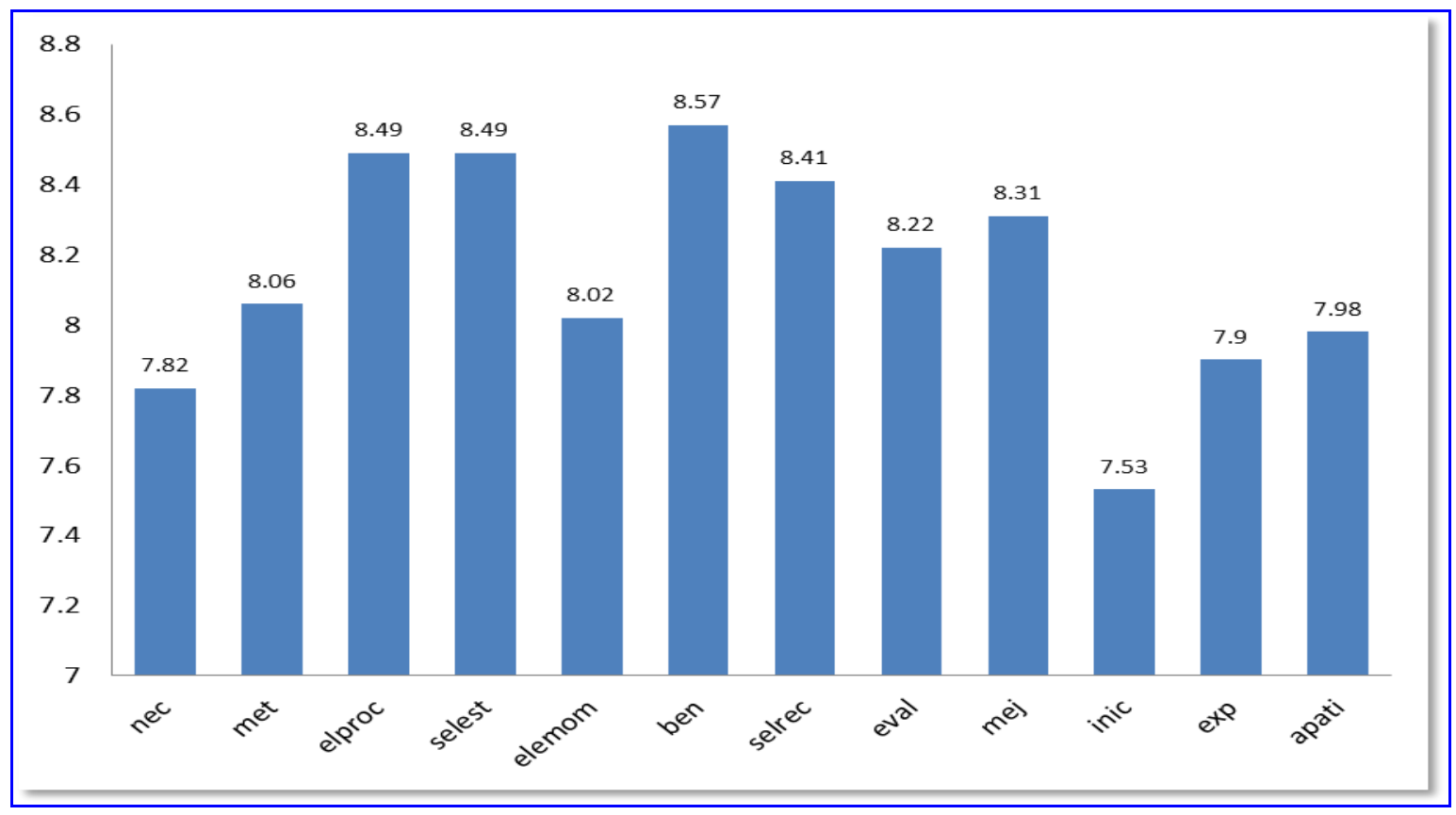

Fuente: Elaboración propia.

En esta agrupación, a partir de la $X x=8.17$, los estudiantes de bachillerato de 15 a 18 años de edad muestran como valor alto $(X=8.57)$ la aceptación del estudiante por reconocer como un gran beneficio el aprender, es decir, le apuesta a la vida académica como medio de obtener a cambio mejores condiciones de vida.

Se muestra que medianamente ha desarrollado habilidades o competencias para elegir los procedimientos y seleccionar sus propias estrategias y recursos, utilizando de manera regularmente aceptable las tecnología para aprender por si solo sin la conducción de un profesor, lo que significa que la adquisición de las características para el aprendizaje autónomo y autodirigido representan un área de oportunidad que puede mejorar si es atendida por el sector educativo.

Por otra parte, el estudiante muestra muy poco $(X=7.53)$ interés por asumir la iniciativa de buscar información y prepararse para un buen desempeño académico, es decir, no se siente lo suficientemente motivado para asumir de manera responsable lo que se le exige, lo que representa una debilidad para desarrollar la competencia del aprendizaje autónomo. 
Para el caso de los jóvenes universitarios cuyas edades van de los 18 a los 23 años, muestran mayor habilidad para trazar metas sobre lo que desean alcanzar con el aprendizaje, se declara con bastante habilidad para elegir por sí mismo los procedimientos que más le funcionan seleccionando sus propios recursos. Sin embargo, muy poco analiza la valoración de su desempeño, declara en el mismo sentido recurrir muy poco al uso de las tecnologías para la realización de sus actividades escolares de manera autónoma.

Se infiere a partir de los resultados que los adolescentes de bachillerato a pesar de que reconocen que el aprender representa la oportunidad para obtener beneficios en la vida, no se sienten lo suficientemente motivados para tomar la iniciativa en la búsqueda de los medios y herramientas que mejor les funcione, lo que implica que en su estilo de aprendizaje no han desarrollado las competencias para el aprendizaje autónomo o autodirigido, por lo que muestran cierta dependencia de la conducción del profesor para sentirse seguros de alcanzar el éxito académico.

Tabla 2. Características del aprendizaje autodirigido en jóvenes universitarios.

\begin{tabular}{|l|c|c|c|c|c|c|}
\hline \multicolumn{1}{|c|}{ VARIABLE } & MIN & MAX & X & S & CV & MO \\
\hline Necesidad de información & 0 & 10 & 8.84 & 1.74 & 0.19 & 10 \\
\hline Metas al estudiar & 2 & 10 & 9.1 & 1.44 & 0.15 & 10 \\
\hline Elección de procedimientos & 4 & 10 & 9 & 1.45 & 0.16 & 10 \\
\hline Selección de estrategias & 4 & 10 & 8.76 & 1.45 & 0.16 & 10 \\
\hline Elección de momentos óptimos & 4 & 10 & 8.56 & 1.34 & 0.15 & 10 \\
\hline Utilidad del aprendizaje & 3 & 10 & 8.7 & 1.56 & 0.17 & 10 \\
\hline Selección de recursos & 4 & 10 & 8.94 & 1.28 & 0.14 & 10 \\
\hline Autoevaluación & 0 & 10 & 8.48 & 1.96 & 0.23 & 10 \\
\hline Mejora del desempeño & 3 & 10 & 8.66 & 1.37 & 0.15 & 10 \\
\hline Iniciativa & 5 & 10 & 8.66 & 1.23 & 0.14 & 10 \\
\hline Aprendizaje mediante las TIC & 0 & 10 & 8.62 & 1.71 & 0.19 & 10 \\
\hline Expectativa & 0 & 10 & 8.26 & 2.08 & 0.25 & 10 \\
\hline \multicolumn{7}{|c|}{ Xs=8.71 8.93} \\
\hline
\end{tabular}

Fuente: Elaboración propia. 
Figura 2. Características del aprendizaje autodirigido en jóvenes universitarios.

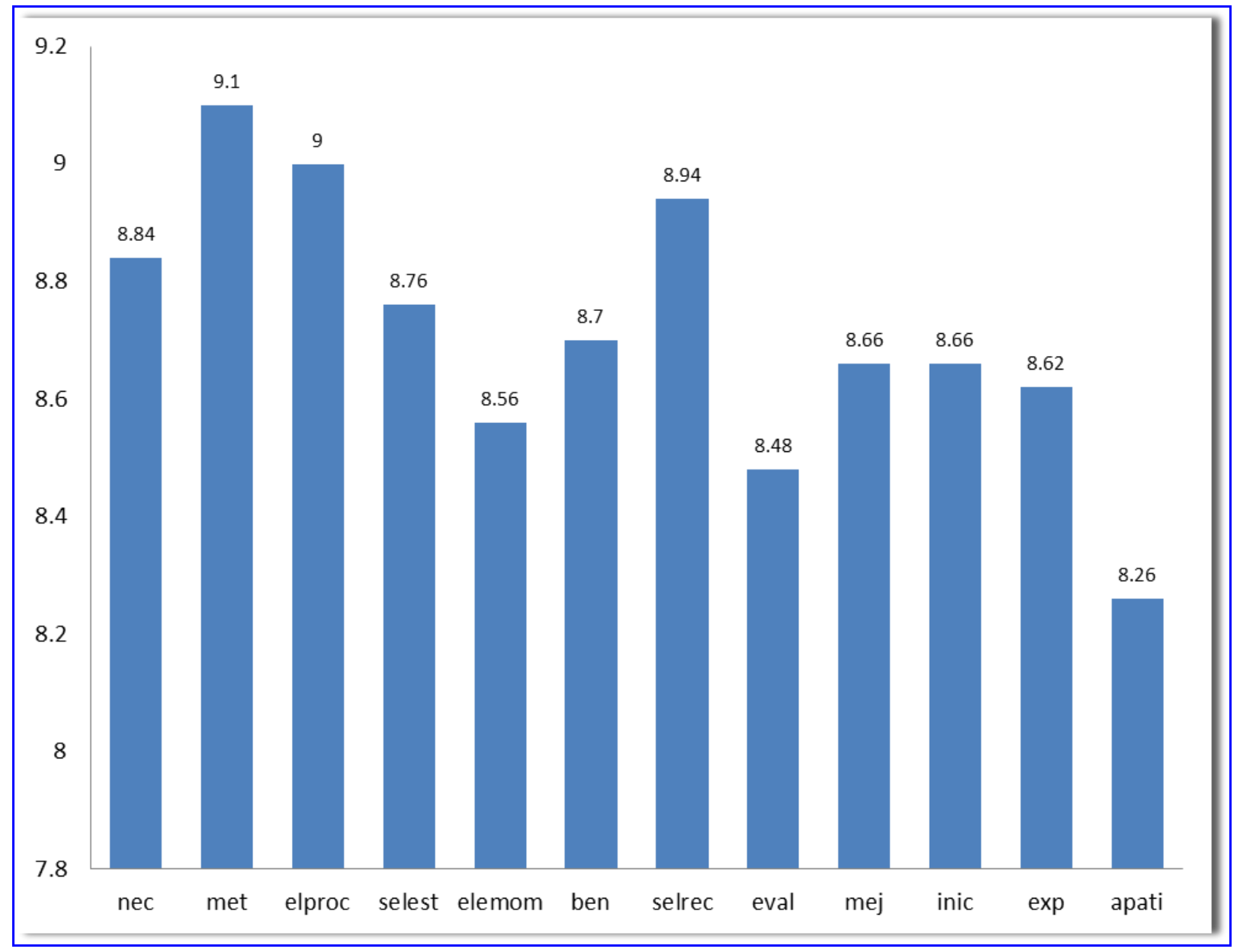

Fuente: Elaboración propia.

En el caso de los jóvenes universitarios de 18 a 23 años, los resultados muestran que tienen más claridad de sus metas escolares, por lo que son más autodirigidos en sus estilos de aprendizaje, sin embargo son poco proclives al uso de las tecnologías para la generación del aprendizaje autónomo. Es decir, los jóvenes del nivel superior tienen claro lo que quieren lograr al estudiar ciertos temas, ellos mismos escogen la mejor forma de aprender y saben seleccionar los mejores recursos para lograr un buen desempeño en la tarea, lo cual nos indica que estos estudiantes poseen un buen nivel de aprendizaje autodirigido, sin embargo, las variables con baja puntuación demuestran que no analizan su desempeño una vez finalizado el trabajo o tarea a realizar, ni usan de manera voluntaria las Tecnologías de la Información y la Comunicación (TIC) para aprender, lo que podría entorpecer el aprendizaje 
autodirigido puesto que es importarte evaluar los resultados siempre para saber qué estrategias metacognitivas volver a aplicar y cuáles no, de esta manera, se mejoraría el proceso del propio aprendizaje. Según Monereo (2002) promover la metacognición implica enseñar a los estudiantes a conocerse mejor como estudiantes, es decir, conducir a que identifiquen sus dificultades, y reconozca sus habilidades y preferencias, en este sentido la autovaloración del desempeño es de vital importancia.

En lo que respecta al aprendizaje colaborativo, esta agrupación, a partir de la $X x=7.79$, los estudiantes de bachillerato muestran que tienen mucha disposición a participar en trabajos colectivos, declaran que se sienten muy aptos para generar un ambiente de comunicación entre sus compañeros en la realización de trabajos colectivos, es decir, los adolescentes de bachillerato prefieren y se sienten hábiles para desempeñarse en equipos de manera colaborativa. 
Tabla 3. Característica del aprendizaje colaborativo en adolescentes de bachillerato.

\begin{tabular}{|l|c|c|c|c|c|c|}
\hline \multicolumn{1}{|c|}{ VARIABLE } & MIN & MAX & X & S & CV & MO \\
\hline Responsabilidad & 1 & 10 & 7.96 & 1.97 & 0.25 & 9 \\
\hline Destreza & 0 & 10 & 7.86 & 1.84 & 0.26 & 9 \\
\hline Actitud & 0 & 10 & 7.82 & 2.36 & 0.30 & 9 \\
\hline Participación & 2 & 10 & 8.37 & 1.78 & 0.21 & 10 \\
\hline Interacción & 1 & 10 & 8.24 & 2.15 & 0.26 & 9 \\
\hline Organización de roles & 0 & 10 & 7.92 & 2.48 & 0.31 & 10 \\
\hline Exposición & 0 & 10 & 7.59 & 2.49 & 0.33 & 8 \\
\hline Concreción & 0 & 10 & 7.45 & 2.95 & 0.40 & 9 \\
\hline Satisfacción & 2 & 10 & 8.33 & 1.83 & 0.22 & 10 \\
\hline Uso de tecnologías & 0 & 10 & 7.73 & 2.22 & 0.29 & 8 \\
\hline Autonomía & 0 & 10 & 7.31 & 2.62 & 0.36 & 8 \\
\hline $\begin{array}{l}\text { Adaptación al uso de plataformas } \\
\text { educativas }\end{array}$ & 0 & 10 & 7.67 & 2.42 & 0.32 & 10 \\
\hline Medios electrónicos & 0 & 10 & 7.88 & 2.59 & 0.33 & 10 \\
\hline Retroalimentación en foros & 0 & 10 & 7.59 & 2.58 & 0.34 & 8 \\
\hline Rol del profesor & 0 & 10 & 7.0 & 3.03 & 0.43 & 8 \\
\hline & Ls=8.16 & Li=7.43 & & \\
\hline
\end{tabular}

Fuente: Elaboración propia.

Figura 3. Características del aprendizaje colaborativo en estudiantes de bachillerato.

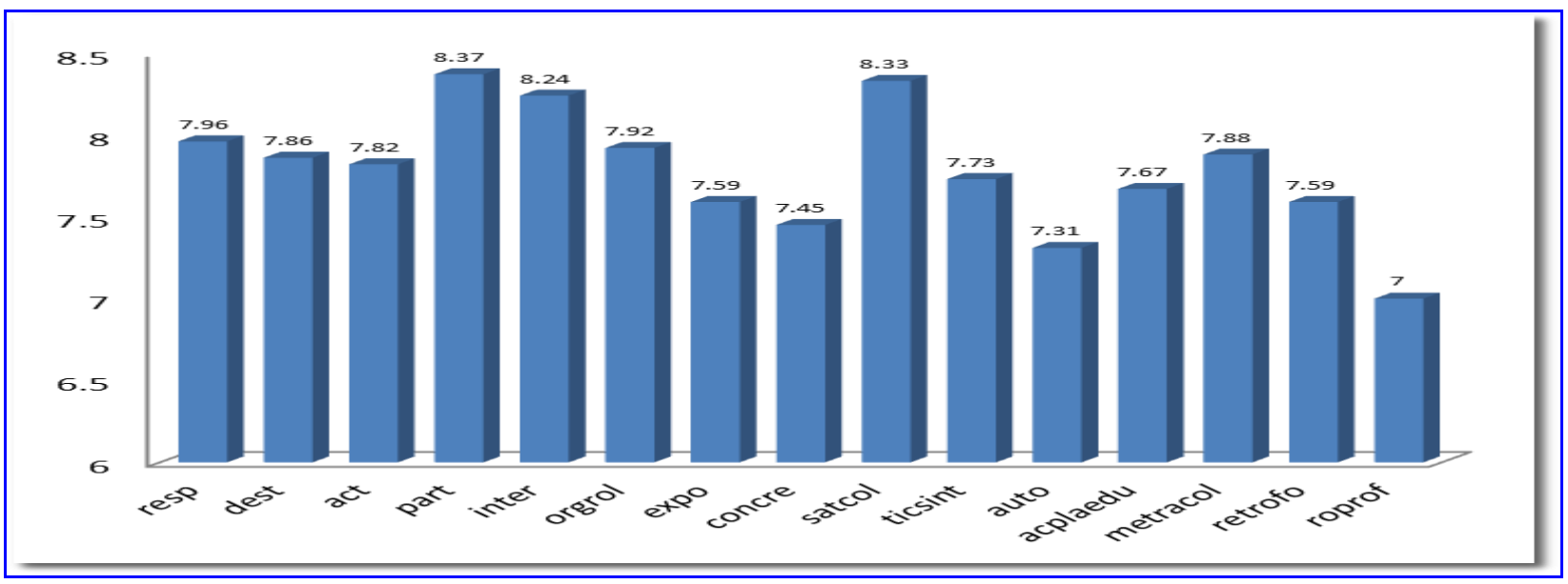

Fuente: Elaboración propia. 
El adolescente de bachillerato se muestra regularmente dispuesto a asumir y responder con asertividad entre sus compañeros, asume a las tecnologías como una herramienta regularmente aceptable para aprender, muestra de manera aceptable su interés para utilizar las plataformas virtuales como método de intervención didáctica, el adolescente de bachillerato usa de manera regular las redes sociales y correos electrónicos para compartir trabajos escolares, por lo que medianamente acepta que aprende trabajando de manera grupal utilizando las TIC. Es decir, se infiere que el adolescente acepta tener contacto con las tecnologías y reconocerlas como medio idóneo para el aprendizaje colaborativo, mostrando cierta disposición, no obstante es un área que puede ser potencializada como parte de un programa escolar.

Por otra parte, el estudiante de bachillerato, considera que en los trabajos colaborativos en espacios virtuales $(X=7.31)$ poco pueden aprender sin la intervención del profesor, por lo que el joven requiere del apoyo del profesor en la conducción de las actividades colaborativas que se realizan de manera virtual y que muy escasamente $(X=7)$ siente la orientación del profesor en los trabajos virtuales.

En los jóvenes universitarios los resultados arrojaron que no hay datos estadísticamente significativos en cuanto a variables con valores altos, lo que implica que aceptan de manera regular todas las características del trabajo colaborativo, es decir, muestran disposición y desempeño si así se les indica trabajar.

Por otra parte, las variables que resultaron más bajas en comparación con la media de medias fueron la aceptación que se tiene hacia las plataformas educativas $(X=7.3)$ y el rol del profesor en los trabajos colaborativos $(X=6.58)$. Por lo que se infiere que demuestran poca habilidad en la utilización de espacios virtuales como forma de trabajo colaborativo, ya que no sienten el apoyo del profesor en estos medios.

A partir de los resultados se infiere que los estudiantes universitarios encuestados se sienten aptos para generar un ambiente de comunicación en trabajos en equipo, lo cual nos proporciona la idea de que, aunque no existe una preferencia a trabajar en grupos colaborativos por encima del trabajo individual, los estudiantes se disponen aceptablemente a trabajar con otros. Por otra parte, podemos inferir que no se sienten seguros en el trabajo 
colaborativo a través de plataformas educativas, ya que no hay una supervisión y participación por parte del docente.

Los resultados demuestran que el papel del docente en los ambientes virtuales es muy importante para generar una nueva cultura en el aprendizaje y promover la relación pedagógica equilibrada que permita la generación y la confianza en el aprendizaje autónomo y colaborativo.

Silva (2010) refiere que para el funcionamiento del espacio virtual de aprendizaje se requiere la importante actuación del tutor "profesor virtual", quien debe mantener vivos los espacios comunicativos, facilitar el acceso a los contenidos, animar el diálogo entre los participantes, ayudarles a compartir su conocimiento y a construir conocimiento nuevo. No se trata entonces de esperar a que de manera espontánea surja la competencia de la autoconducción en el proceso de aprender en la interactividad, se trata de dinamizar las potencialidades individuales y fomentar la interacción regulada. Se incluye entre sus habilidades y competencias requeridas la de ser acompañante y facilitador en el acceso y motivación para la inclusión en estos nuevos espacios. El aprendizaje virtual requiere un profesor que se entrene previamente, para que con una base pedagógica, priorice las bondades de la interacción, facilite el ingreso al estudiante en el proceso digital y su adaptación a que a través de la estrategia digital aprenda y acepte la nueva dinámica normativa.

\section{Las estrategias de aprendizaje en entornos virtuales en los jóvenes}

\section{estudiantes}

El diseño y la implementación de estrategias didácticas tanto en la educación presencial como en la creación de entornos virtuales son fundamentales para el logro de las condiciones que permitan la activación del proceso cognitivo, por ello han de estar bien sustentadas en un enfoque pedagógico, lograr la motivación, la participación, el interés y la conformación de metas e intenciones académicas marca la diferencia en el logro del aprendizaje autónomo y significativo. 
Sierra (2011) refiere a la autorregulación como competencia que incluye que el individuo conozca su propio proceso de aprendizaje, programe conscientemente sus estrategias de aprendizaje, de memoria, de solución de problemas y toma de decisiones, y se expresa como autonomía al aprender.

En esta agrupación de variables se caracteriza a los jóvenes con respecto a su habilidad para el manejo de estrategias virtuales, describen así la manera en que los jóvenes diseñan sus métodos para acercarse a la información y al procesamiento cognitivo de la misma.

A partir de los valores de la $(X=9.09)$ para esta agrupación, se muestra que los adolescentes de bachillerato son hábiles para encontrar información que les es muy útil en Internet $(X=9.49)$, declaran sentirse con un manejo aceptable en cuanto a la organización, selección y tiempos eficientes en su búsqueda de información. Sin embargo muestran menos interés $(X=8.62)$ por buscar diferentes puntos de vista para confrontar la información que requieren, es decir, una vez que localizan la información aceptan sin contrastar la diversidad de opiniones de los distintos autores.

Para el caso de los jóvenes universitarios esta agrupación no mostró niveles altos en sus respuestas, declaran lograr de manera regular tanto el acercamiento como la organización, selección y análisis de la información, sin embargo una de sus debilidades es la eficiencia en la administración de sus tiempos de búsqueda, ya que declaran tener poca habilidad $(X=6.96)$ para encontrar rápidamente la información.

En cuanto a las formas de aprendizaje y el acercamiento al conocimiento, los estudiantes de bachillerato declaran que sin la presencia del profesor sienten mucha necesidad $(X=9.18)$ de diseñar una estrategia, aunque se sienten muy hábiles $(X=9.10)$ en la planificación de sus tiempos. A pesar de que declaran saber autoconducirse de manera regular en la dinámica de los entornos virtuales, demuestran muy poca preferencia $(X=7.21)$ por los cursos con apoyo virtual.

Los jóvenes universitarios mayores demuestran por su parte que pueden distinguir muy fácilmente $(X=8.74)$ la utilidad y validez de la información obtenida y de manera muy escasa $(X=6.76)$ sienten necesidad de la orientación del profesor para buscar la información para la tarea solicitada, es decir, son más independientes en la realización de sus actividades, sin 
embargo, al igual que los jóvenes de bachillerato demuestran poca preferencia $(X=5.06)$ por los apoyos virtuales como estrategia de aprendizaje.

En ambas poblaciones se reconoce el desarrollo de algunas destrezas y competencias para el aprendizaje autónomo y para el desempeño del trabajo colaborativo en medios virtuales, sin embargo, también se muestra que son habilidades que no se han potenciado lo suficiente desde el ámbito educativo, desaprovechando las características que como nativos digitales poseen las nuevas generaciones y por ende el acercamiento natural que los más jóvenes han venido realizando con los alcances tecnológicos, en el caso de los adolescentes mayores en etapa universitaria, se demuestra la falta de acompañamiento del profesor tutor en los ambientes virtuales.

\section{Discusión}

A partir de las bondades de las innovaciones tecnológicas, se presenta ante el ámbito educativo la oportunidad de avanzar a un mejor ritmo y con mayor amplitud y cobertura que la enseñanza tradicional, sin embargo, esto exige posicionarse desde una perspectiva teórica metodológica que nos permita una visión más activa y crítica en la construcción de los saberes por parte de quien aprende. Sin lugar a dudas la creación de espacios virtuales resulta una estrategia atractiva para fomentar en el alumnado una serie de características deseables en el nuevo perfil; mediante estrategias adecuadas a los entornos digitales se puede estimular el pensamiento crítico ya que se presenta un análisis basado en al contrastación de la información, información que puede ser aprendida más eficientemente que la enseñanza tradicional; se ha comprobado que la combinación de canales sensitivos mediante la utilización de gráficos, animaciones, sonidos entre otros, incita a la comprensión activa de manera más natural.

Por otra parte, se ofrecen mejores condiciones para el aprendizaje cooperativo, ya que el hecho de permanecer juntos no es el requisito indispensable, sino la habilidad de interacción y negociación cognitiva la que permea en el trabajo colaborativo y ésta es factible en el diseño de estrategias digitales, sin considerar la permanencia física extendiéndola inclusive a poblaciones o grupo mayores conformando comunidades de aprendizaje, en términos generales la computadora deja de verse como un instrumento lineal y personal para verse 
como una herramienta que amplía las potencialidades en la interacción, donde lo más importante es la actividad cognitiva que el sujeto que aprende puede realizar. Para Rotstein (2006) la colaboración como modalidad pedagógica, la participación y los intercambios en las aulas tanto físicas como virtuales se produce a través de los pares. La discusión y el dialogo se constituyen en escenarios de aprendizaje y no solo en dispositivos para la apropiación de conocimientos, porque para que la interacción con otros posibilite avances para el aprendizaje se requiere la comprensión conjunta de lo que se aborda, esto implica una visión compartida.

Según Martínez (2008) todo trabajo colaborativo es un trabajo de grupo, pero no todo trabajo de grupo es un trabajo colaborativo. El trabajo colaborativo es la conformación de un grupo de sujetos con conocimientos similares en el tema, donde no surge un líder como en un trabajo de grupo normal, por el contrario, el liderazgo es compartido por todos los integrantes de esta "comunidad" así como la responsabilidad del trabajo y/o el aprendizaje. Se desarrolla entre los integrantes de dicho equipo, el concepto de ser mutuamente responsables del aprendizaje de cada uno de los demás.

Es a través de la exploración y la libre indagación que el estudiante desarrolla el aprendizaje autónomo, planifica y controla desde sus propias posibilidades y recursos.

Sin embargo, es imprescindible que para que los objetivos sean alcanzados en su intencionalidad se generen condiciones que de alguna manera permitan la instalación de una cultura más abierta sobre el aprendizaje. Una de las principales condiciones y retos es poner al alcance de los jóvenes, las herramientas a las que difícilmente ellos podrían acceder, háblese de infraestructura y servicios, pero más aún del capital humano docente habilitado para que asuma un nuevo rol como facilitador.

Como los datos obtenidos en este estudio lo demuestran, uno de los puntos débiles en el diseño y creación de ambientes colaborativos como parte de las estrategias en espacios virtuales es la falta de orientación pertinente por parte del docente, que también se enfrenta al reto de romper con las barreras culturales.

Cabello y Renzo (2013) demuestran en su estudio que más de la mitad de la población docente sabe usar, usa y además, tiene computadora en el hogar, constituye un rasgo típico 
sobre todo en el grupo de maestros de mayor edad y antigüedad, ya que los docentes jóvenes por lo general no poseen computadora e igualmente se consideran usuarios efectivos y competentes para operarla.

Las representaciones que los docentes tienen acerca de su vínculo con la tecnología varían según la edad, pero esto ocurre en un contexto en el que saber y usar la Computadora Personal (PC por sus acrónimo en inglés) es sinónimo de saber y usar el procesador de textos. La mayor parte de los docentes han sido formados para realizar su práctica en esquemas áulicos, sin embargo, aún la capacitación en los nuevos enfoques innovadores no contempla todos los factores que habiliten el diseño de estrategias virtuales las cuales permitan al docente el cambio de rol, en estas condiciones el acompañamiento como tutor virtual se convierte en una barrera complicada de romper.

Isianny (2011) comenta en su trabajo con respecto a la escasez de docencia habilitada en la intervención en entornos virtuales, a nivel mundial, sólo un tercio de profesores que dictan clases virtuales han sido entrenados para enseñar por Internet.

Esta es una de las principales razones por las que los entornos virtuales no logran la motivación suficiente en el estudiante pues sin un profesor convencido y habilitado, el estudiante no encuentra ese punto de donde afianzarse para continuar en la exploración y la controversia de la que se aprende en la sociedad de la información. Segura y Gallardo (2013) atribuyen a un desconocimiento o malas competencias tecnológicas, tanto por parte del alumnado como del profesorado como causa principal del rechazo a la incorporación de estos medios.

El riesgo de confundir el uso per se de estos implementos tecnológicos como una acción innovadora y pedagógica puede engañar al objetivo y meta educativa. Salmerón (2010) advierte que la intervención de los espacios virtuales solo adquiere valor pedagógico cuando las interpretamos como artefactos mediadores entre el docente y el alumnado o entre iguales, es decir, en la interacción mediada para la construcción del conocimiento a partir de la actividad directa del sujeto que aprende con la ayuda del profesor.

Para Villarruel (2009), para poder alcanzar el dominio de la autonomía en el aprendizaje se requiere de un alto grado de autocontrol y autorregulación, el cual sólo puede alcanzarse a 
través de estrategias que lleven al estudiante a desarrollar un pensamiento crítico y creativo, donde se aprecien las habilidades de una persona reflexiva, en ella va incluida la identificación de necesidades y habilidades educativas o escolares.

Los resultados demuestran que hay potencial en las dos poblaciones analizadas para desempeñarse en los espacios virtuales, sin embargo, este estudio se limitó a considerar como sujeto de estudio solamente a la población estudiantil, sin tomar en cuenta que el fenómeno de estudio incluye al docente como un elemento importante por su compromiso que como actor educativo le corresponde en la consolidación de las condiciones necesarias para la creación de espacios innovadores. Por lo tanto, este estudio establece un punto de partida para nuevas líneas de investigación que incluyan al docente en lo referente al diseño de estrategias que integren herramientas tecnológicas innovadoras.

\section{Conclusiones}

Los adolescentes de bachillerato a pesar de que reconocen que el aprender representa la oportunidad para obtener beneficios en la vida, no se sienten lo suficientemente motivados para tomar la iniciativa en la búsqueda de los medios y herramientas que mejor les funcione, lo que implica que en su estilo de aprendizaje no han desarrollado las competencias para el aprendizaje autónomo o autodirigido, por lo que muestran cierta dependencia de la conducción del profesor para sentirse seguros de alcanzar el éxito académico.

El adolescente acepta tener contacto con las tecnologías y reconocerlas como medio idóneo para el aprendizaje colaborativo, mostrando cierta disposición, sin embargo es un área que puede ser potenciada como parte de un programa escolar.

Los estudiantes universitarios se sienten aptos para generar un ambiente de comunicación en trabajo en equipo, lo cual nos proporciona la idea de que, aunque no existe una preferencia a trabajar en grupos colaborativos por encima del trabajo individual, los estudiantes se disponen aceptablemente a trabajar con otros. Por otra parte, podemos inferir que no se sienten seguros en el trabajo colaborativo a través de plataformas educativas, ya que no hay una supervisión y participación por parte del docente. 
Los resultados demuestran que el papel del docente en los ambientes virtuales es muy importante para generar una nueva cultura en el aprendizaje y promover la relación pedagógica equilibrada que permita la generación y la confianza en el aprendizaje autónomo y colaborativo.

Los adolescentes de bachillerato son hábiles para encontrar información que les es muy útil en Internet, sin embargo, muestran menos interés por buscar diferentes puntos de vista para confrontar la información que requieren, es decir, una vez que localizan la información la aceptan sin contrastar la diversidad de opiniones de los distintos autores por lo que no logran hacer un proceso de análisis. Mientras que para los jóvenes universitarios una de sus debilidades es la eficiencia en la administración de sus tiempos de búsqueda, ya que declaran tener poca habilidad para encontrar rápidamente la información.

En ambas poblaciones se reconoce el desarrollo de algunas destrezas y competencias para el aprendizaje autónomo y para el desempeño del trabajo colaborativo en medios virtuales, sin embargo, también se muestra que son habilidades que no se han potenciado lo suficiente desde el ámbito educativo, desaprovechando las características que como nativos digitales poseen las nuevas generaciones y por ende el acercamiento natural que los más jóvenes han venido realizando con los alcances tecnológicos, en el caso de los adolescentes mayores en etapa universitaria, se demuestra la falta de acompañamiento del profesor tutor en los ambientes virtuales. 


\section{Bibliografía}

Alcalde, I. (2015). El Trabajo Colaborativo en entornos virtuales. Disponible en: http://www.ignasialcalde.es/el-trabajo-colaborativo-en-entornos-virtuales/

Ballenato, G. (2009). Estrategias de aprendizaje en un entorno virtual. IV Jornada de Innovación Pedagógica del Proyecto ADA- Madrid.

Bransford, J., Brown, A., Coking, R. (1999). La creación de ambientes de aprendizaje en la escuela. Serie Cuadernos de la Reforma, México: Secretaría de Educación Pública.

Bello, R. (2005). Educación Virtual: Aulas sin Paredes. Disponible en: http://www.educar.org/articulos/educacionvirtual.asp

Cabello R., Renzo M. (2013). TIC y EDUCACIÓN: Competencias Tecnológicas y capacitación para la apropiación de las tecnologías. Disponible en: http://razonypalabra.org.mx/anteriores/n49/bienal/Mesa\%2013/PonenciaRoxanaCab ello2.pdf

Gross, B., (2002). Constructivismo y diseños de entornos virtuales de aprendizaje. Revista de Educación. No. 328.

Jhonson, D., Jhonson, R., Holubec, E. (1999). El aprendizaje cooperativo en el aula. Buenos Aires. Editorial Paidos.

Martínez, F., Prendes, M. (2008). Estrategias y Espacios Virtuales de Colaboración para la Enseñanza Superior. Revista Internacional de Ciencias Sociales y Humanidades, SOCIOTAM. XVIII (2).

Monereo, C., Duran, D. (2002). Entramados. Métodos de enseñanza cooperativa y colaborativa. Barcelona: Edebé.

Prensky, M. (2001, 09). Digital Natives, Digital Immigrants Part 1. On the Horizon, 9(5), 1 6. doi:10.1108/10748120110424816. 
Roinstein, B., Sainz C., Scassa, A., Simesen, A. (2006). El trabajo colaborativo en entornos virtuales de aprendizaje. Revista Cognición, No. 7. Pags 38-45.

Sierra, I. (2011). Concepciones y Estrategias Docentes en Ambientes Virtuales. Serie Aprender a Educar. Colombia.

Silva, J. (2010). El rol del tutor en los entornos virtuales de aprendizaje. Innovación Educativa, vol. 10, núm. 52, pp. 13-23 Instituto Politécnico Nacional Distrito Federal, México

Salmerón, H., Rodríguez, S., Gutiérrez, C. (2010). Metodologías que optimizan la comunicación en entornos de aprendizaje virtual. Revista Comunicar, núm. 34 163171. España.

Villarruel M. (2009). La práctica educativa del maestro mediador. . Instituto Tecnológico Úrsulo Galván, México. SEP-DGEST. Revista Iberoamericana de Educación. Edit. Organización de Estados Iberoamericanos para la Educación, la Ciencia y la Cultura (OEI) 